\title{
Comment on the first-order Fermi acceleration at ultra-relativistic shocks
}

\author{
M. Ostrowski ${ }^{1,2}$ and J. Bednarz ${ }^{1}$ \\ 1 Obserwatorium Astronomiczne, Uniwersytet Jagielloński, ul. Orla 171, 30-244 Kraków, Poland \\ 2 Institut für Theoretische Physik, Lehrstuhl IV: Weltraum und Astrophysik, Ruhr-Universität Bochum, Germany
}

Received 17 April 2002 / Accepted 23 July 2002

\begin{abstract}
The first-order Fermi acceleration process at an ultra-relativistic shock wave is expected to create a particle spectrum with the unique asymptotic spectral index $\sigma_{\gamma \gg 1} \approx 2.2$. Below, we discuss this result and differences in its various derivations, which - explicitly or implicitly - always require highly turbulent conditions downstream of the shock. In the presence of medium amplitude turbulence the generated particle spectrum can be much steeper than the above asymptotic one. We also note problems with application of the pitch angle diffusion model for particle transport near the ultra-relativistic shocks.
\end{abstract}

Key words. acceleration of particles - shock waves - X-rays: bursts - gamma-rays: bursts - ISM: cosmic rays

\section{Introduction}

Ultra-relativistic shock waves suggested to be sources of gamma-ray bursts are also expected by some authors to produce ultra-high-energy cosmic ray particles. A process of the first-order Fermi acceleration in such shocks was discussed in a series of papers by Bednarz \& Ostrowski $(1997,1998 ;$ see also Bednarz 2000a,b), Gallant \& Achterberg (1999; see also Achterberg et al. 2001), Kirk et al. (2000) and Vietri (2002). Below, in Sect. 2, we briefly compare and discuss different approaches to the considered acceleration process, leading to the asymptotic spectral index $\sigma_{\gamma \gg 1} \approx 2.2$. We note an important fact that in order to derive this result, essentially all these studies consider the large amplitude magnetic field perturbations near the shock, with the turbulence power concentrated in the short wavelength range. The particle energy spectra generated in shocks propagating in a mildly turbulent medium, with the limited turbulence downstream of the shock, can be much steeper in ultra-relativistic shocks than the above "asymptotic" one.

In the discussion below we neglect the strictly parallel shocks, where some of our objections can be invalid. However, such shocks are not expected to frequently occur in the universe.

\section{On the first-order Fermi acceleration at ultra-relativistic shocks}

The first-order Fermi acceleration process at an ultrarelativistic shock wave involves extreme particle anisotropy at the shock in the upstream plasma rest frame (UPF), and more

Send offprint requests to: M. Ostrowski, e-mail: mio@oa.uj .edu.pl mild distributions in the shock normal rest frame or the downstream plasma rest frame (cf. Begelman \& Kirk 1990). Let us consider an individual cosmic ray particle acceleration starting with a particle crossing the shock upstream (cf. a detailed discussion by Gallant \& Achterberg 1999). Then, in UPF, its momentum is nearly parallel to the shock normal. When the shock Lorentz factor is large $(\gamma \gg 1)$ the particle moves in front of the shock for a time required for a slight deflection of its momentum allowing the shock to overtake it and transmit to the downstream region. The deflection proceeds due to the magnetic field upstream of the shock, consisting of the large scale smooth background structure perturbed by the MHD fluctuations. This tiny change of particle momentum upstream of the shock allows for its transmission downstream of the shock, where - due to the Lorentz transformation with a large $\gamma-$ its momentum direction can be changed at a large angle with respect to its original direction before the transmission upstream. Such large amplitude angular scatterings can enable a finite fraction of particles to follow trajectories leading to the successive transmissions upstream of the shock. Repeating of the described loops, with each roughly doubling the particle energy, leads to formation of the power law particle spectrum. Several authors (Bednarz \& Ostrowski 1998; Gallant \& Achterberg 1999; Gallant et al. 1999) discussed this process leading to formation of the spectrum with the energy spectral index $\sigma \approx 2.2$ at $\gamma \gg 1$. Essentially the same results were obtained within different approaches presented by the above authors and by Kirk et al. (2000) and Vietri (2002). We do not consider here the papers postulating the acceleration process, but not discussing details of the proposed mechanism.

The work of Bednarz \& Ostrowski $(1997,1998)$ was based on Monte Carlo simulations of particle transport governed by small amplitude pitch angle scattering. Thus, depending on the 
scattering parameter $\Delta t$ (a mean time between successive scattering acts) and $\Delta \Omega_{\max }$ (the maximum angular scattering amplitude), we were able to model situations with different mean field configurations and different amounts of turbulence. One should note that the mean field configuration downstream of the shock was derived here from the mean upstream field using the appropriate jump conditions and trajectories of particles interacting with the shock discontinuity were derived exactly for such fields. A particle trajectory was derived in the respective local plasma rest frame, with the Lorentz transformation applied at each particle shock crossing. The approach takes into account correlations in the process due to the regular part of the magnetic field, but irregularities responsible for pitch angle scattering are introduced at random. In order to model particle pitch angle diffusion upstream of the shock, with nearly a delta-like angular distribution $\theta \sim \gamma^{-1}(\theta-$ a momentum vector inclination to the shock normal), an extremely small scattering amplitude should be used ${ }^{1}, \Delta \Omega_{\max } \ll \gamma^{-1}$. Increasing the shock Lorentz factor results in decreasing the momentum perturbation required for its transmission downstream and leaves a shorter time for this perturbation, $t_{1}$. In the applied pitch angle diffusion approach, the momentum variation due to the regular component of the magnetic field scales like $t_{1}$, whence the diffusive change scales like $t_{1}^{1 / 2}$. Thus growing $\gamma$ leads to decreasing $t_{1}$ and the diffusive term has to dominate at sufficiently large $\gamma$. It is the reason why in our simulations the orientation of the regular magnetic field ceases to play a role in the limit $\gamma \rightarrow \infty$, resulting in the spectral index convergence to its asymptotic value.

However, one should note that with decreasing $\Delta t$ and $\Delta \Omega_{\max }$, when the interaction proceeds at the sub-resonance $\left(\ll r_{\mathrm{g}}\right)$ spatial scale, a serious physical problem with the applied approach appears. In order to scatter particle momentum uniformly within a narrow cone centred on the initial momentum, it requires the short wave turbulence to be non-linear at the shortest scales. In our discussion of the "effective" magnetic field, $B_{\mathrm{e}}$, in the pitch angle diffusion simulations (Bednarz \& Ostrowski 1996; cf. Appendix below) we evaluate the lower limit of such an effective field from the curvature of simulated particle trajectories as

$$
B_{\mathrm{e}}=B_{\mathrm{o}} \sqrt{1+\left(0.67 \frac{\Delta \Omega_{\mathrm{max}}}{\Delta t}\right)^{2}},
$$

1 The condition $\Delta \Omega_{\max } \ll \gamma^{-1}$ lead to the excessive computation times. Thus, in our simulations we used a relatively "large" maximum amplitude $\Delta \Omega_{\max }=\frac{1}{2} \gamma^{-1}$, but comparison to the results obtained with smaller $\Delta \Omega_{\max }$ revealed only insignificant differences in the obtained particle spectra (as measured at the escape boundary placed at $4 r_{\mathrm{g}}$ downstream of the shock; $r_{\mathrm{g}}$ is a particle gyroradius). One should note that due to the above choice of $\Delta \Omega_{\max }$ and because in our numerical code the relative particle velocity with respect to the shock instead of the velocity in the plasma rest frame was improperly used for the particle weighting function in the plasma rest frame, the angular distributions presented by us (Bednarz \& Ostrowski 1998) are slightly different in comparison to the results of Gallant et al. (1999) and Kirk et al. (2000). However, this error leads only to a wrongly presented angular distributions and it does not influence particle distributions considered in simulations and the derived spectra. taking into account both the background uniform field $B_{\mathrm{o}}$ and the turbulent component evaluated with the use of the scattering parameters $\Delta \Omega_{\max }$ and $\Delta t$ (in this expression $\Delta t$ is given in angular units, it stands for $\left.c \Delta t / r_{\mathrm{g}}\left(B_{\mathrm{o}}\right)\right)$. Assuming the constant pitch angle diffusion coefficient $\left(\propto\left(\Delta \Omega_{\max }\right)^{2} / \Delta t\right)$ for a series of computations involving smaller and smaller $\Delta \Omega_{\max } \sim \gamma^{-1}$ we had to use $\Delta t$, which scales like $\gamma^{-2}$. As a result, to be consistent with the assumed scattering model, for large shock Lorentz factors the effective magnetic field increases to large values due to the required growing power being concentrated in the short wave turbulence, $B_{\mathrm{e}} \propto \gamma B_{\mathrm{o}}$. Such conditions seem to be unrealistic at least upstream of the shock.

An analogous pitch angle diffusion modelling appended the considerations of Gallant et al. (1999; for a more detailed description see Achterberg et al. 2001). They considered the highly turbulent conditions near the shock leading to the particle pitch angle diffusion with respect to the shock normal, i.e. the regular part of the magnetic field was neglected. These computations gave essentially the same spectral indices as the asymptotic one derived by Bednarz \& Ostrowski (1998). Also, in a variant of this model with uniform magnetic field upstream of the shock and fully chaotic turbulent field downstream, the resulting spectral index did not vary substantially. The physical content of the discussed model is substantially different from the Bednarz \& Ostrowski one because it neglects the influence of the uniform field (or long wavelength perturbations with $\lambda>r_{\mathrm{g}}$ ) resulting in magnetic field correlations at both sides of the shock. Thus, it provides spectra with the asymptotic spectral index at quite moderate $\gamma \sim 10$, a feature also present in the Bednarz \& Ostrowski simulations for parallel shocks. However, if the amplitude of the magnetic field turbulence is limited, these simulations cannot reproduce spectrum steepening (or flattening at intermediate Lorentz factors) in the presence of oblique magnetic fields (cf. Ostrowski 1993; Bednarz \& Ostrowski 1998; Begelman \& Kirk 1990). Both the above models describe essentially the same physical situation for shocks propagating in the highly turbulent medium and, of course, in rarely - if ever - occurring parallel relativistic shocks.

An alternative discussion of the acceleration process presented by Gallant \& Achterberg (1999) was based on a simple turbulence model. In their approach a highly turbulent magnetic field configuration was assumed upstream and downstream of the shock, idealized as cells filled with randomly oriented, uniform (within a cell) magnetic fields. With such an approach, particles crossing the shock enter a new cell with a randomly selected magnetic field configuration. Thus, there always occur configurations allowing some particles crossing downstream to reach the shock again and again. As a result of successive energy increases of the same finite fraction of accelerated particles, the power law spectrum is formed. In this model there is no need for upstream magnetic field perturbations if the considered oblique magnetic field configuration can turn all upstream particles back to the shock.

Two quasi-analytic approaches to the considered acceleration process were presented by Kirk et al. (2000) and Vietri (2002). Both provide methods to solve the Fokker-Planck equation describing particle advection with the general plasma flow 
and the small amplitude scattering of particle pitch angle as measured with respect to the shock normal. The important work of Kirk et al. modified the Kirk \& Schneider (1987) series expansion approach to treat the delta-like angular distribution upstream of the shock. An analytically more simple Vietri approach applies convenient ansatz'es for the anisotropic upstream and downstream particle distributions, resembling the Peacock (1981) approach to acceleration at "ordinary" relativistic shocks. Both methods confirm the results of the earlier numerical modelling. A deficiency of the above semi-analytic approaches is its inability to treat situations with mildly perturbed magnetic fields, on average oblique to the shock normal. If considered valid for different configurations of the mean magnetic field, these models require the large amplitude short wave turbulence to remove signatures in particle trajectories of the uniform background field or of the long wave perturbations. Thus it provides an alternative description of the same physical situation discussed earlier with numerical methods by Bednarz $\&$ Ostrowski (1998) in the $\gamma \rightarrow \infty$ limit or their parallel shock results and all other authors applying small amplitude pitch angle scattering simulations at parallel shock waves.

\section{Conclusions}

The discussed approaches to the cosmic ray first-order Fermi acceleration at relativistic shocks yield consistent estimates of the asymptotic spectral index $\approx(2.2,2.3)$. However, the result is not as universal as one could infer from the convergent conclusions of different authors, because all presented derivations require (explicitly or implicitly) large amplitude MHD turbulence near the shock. Only the Bednarz \& Ostrowski (1998) modelling allows one to treat - in a simplified way - conditions with medium amplitude perturbations of the magnetic field. In such conditions particle spectra are expected to be very steep at high shock Lorentz factors. The spectra considered by Bednarz \& Ostrowski flatten at large $\gamma$ due to an implicit increase of the short wave turbulence in their model, approaching closer and closer the parallel shock configuration considered by the other authors. Until now the situation with the medium amplitude turbulence, $\delta B<B_{\mathrm{o}}$, has not been studied in the limit of large $\gamma$, however, from comparison with the results of Begelman \& Kirk (1990), Ostrowski (1993) and of Bednarz \& Ostrowski (1998) for intermediate shock Lorentz factors, we expect very steep spectra to be formed in such conditions. Thus, if the conditions with limited turbulence are met at a large $\gamma$ shock, it can be unable to accelerate particles to very high energies in the first-order Fermi mechanism. On the other hand the "low" energy electrons radiating from ultra-relativistic shocks could be accelerated by the non-first-order processes, analogous to the ones discussed by Hoshino et al. (1992) or Pohl et al. (2001).

The main deficiency of the approaches applying the pitch angle diffusion equation, in particular of our own attempt to discuss cases with oblique background magnetic fields, is their limitation to particular, highly turbulent conditions near the shock. This limitation may be significant or non-significant, depending on whether such conditions exist downstream of the ultra-relativistic shock. In the process discussed by
Medvedev \& Loeb (1999) such short-wave non-linear turbulence is created downstream of the shock, in the non-resonant wave-vector range for the shock accelerated particles.

Acknowledgements. MO is grateful to Reinhard Schlickeiser for the invitation to the Institute for Theoretical Physics of the Ruhr University, where this work was partly done, and to Yves Gallant and Bohdan Hnatyk for valuable discussions. The work was supported by the Komitet Badan Naukowych through the grant PB 258/P03/99/17.

\section{Appendix: Evaluation of the effective magnetic field $B_{\mathrm{e}}$ in a numerical code applying the discrete small-amplitude pitch angle scattering method}

Let us evaluate the magnetic field components responsible for regular, $\Delta \theta_{\mathrm{r}}$, and turbulent, $\Delta \theta_{\mathrm{t}}$, angular deviations of the particle momentum during a single particle propagation time-step $\Delta t$. For the regular deviation due to the mean magnetic field $B_{\mathrm{o}}$

$\Delta \theta_{\mathrm{r}} \sim \frac{c \Delta t}{r_{\mathrm{g}}\left(B_{\mathrm{o}}\right)}=\frac{e}{p} B_{\mathrm{o}} \Delta t$

where $r_{\mathrm{g}}(B)=e B /(p c), p$ is the relativistic particle momentum and $e$ its charge. In the above estimate the undefined proportionality factor depends on local particle distribution anisotropy. For the turbulent component of the magnetic field one can evaluate its lower limit analogously to (A.1) by assuming the turbulent field component, $B_{\mathrm{t}}$, to be uniform at the spatial scale $c \Delta t$ :

$\Delta \theta_{\mathrm{t}} \sim \frac{c \Delta t}{r_{\mathrm{g}}\left(B_{\mathrm{t}}\right)}=\frac{e}{p} B_{\mathrm{t}} \Delta t$

For the considered model involving uniform scattering within a narrow cone of the opening angle $\Delta \Omega_{\max } \ll 1$, the mean scattering angle equals $\frac{2}{3} \Delta \Omega_{\max }$. If the magnetic field components $\boldsymbol{B}_{\mathrm{o}}$ and $\boldsymbol{B}_{\mathrm{t}}$ are oriented randomly with respect to each other, then the effective field modifying particle trajectory, $B_{\mathrm{e}}$, can be evaluated as $B_{\mathrm{e}}^{2}=B_{\mathrm{o}}^{2}+B_{\mathrm{t}}^{2}$. With the estimate $\frac{e}{p} B_{\mathrm{t}} \Delta t=\frac{2}{3} \Delta \Omega_{\max }$ one obtains $B_{\mathrm{t}}=B_{\mathrm{o}}\left\{\frac{2}{3} \Delta \Omega_{\max } /\left[\left(e B_{\mathrm{o}} / p\right) \Delta t\right]\right\}$ and he can evaluate $B_{\mathrm{e}}$ as

$B_{\mathrm{e}}=B_{\mathrm{o}} \sqrt{1+\left[\frac{2}{3} \frac{\Delta \Omega_{\max }}{\frac{e B_{\mathrm{o}}}{p} \Delta t}\right]^{2}}$

In this rough estimate we give the lower contribution from the irregular magnetic field by assuming that trajectory perturbations are due to structures of the wavelength $\lambda \sim c \Delta t$. In the case of turbulence power concentrated at shorter waves the required wave power is even higher to cause the considered scattering. The turbulence power with $\lambda>c \Delta t$ would provide correlations of successive scatterings, excluded in the considered model. 


\section{References}

Achterberg, A., Gallant, Y. A., Kirk, J. G., \& Guthmann, A. W. 2001, MNRAS, 328, 393

Bednarz, J. 2000a, Ph.D. Thesis, Jagiellonian University [astro-ph/0005075]

Bednarz, J. 2000b, MNRAS, 315, L37

Bednarz, J., \& Ostrowski, M. 1996, MNRAS, 283, 447

Bednarz, J., \& Ostrowski, M. 1997, in Proc. 25th Int. Cosmic Ray Conf. (Durban), OG 9.1.6

Bednarz, J., \& Ostrowski, M. 1998, Phys. Rev. Lett., 80, 3911

Bednarz, J., \& Ostrowski, M. 1999, MNRAS, 310, L11

Begelman, M., \& Kirk, J. G. 1990, ApJ, 353, 66

Gallant, Y. A, \& Achterberg, A. 1999, MNRAS, 305, L6
Gallant, Y. A, Achterberg, A., \& Kirk, J. G. 1999, A\&AS, 138, 549

Hoshino, M., Arons, J., Gallant, Y. A., \& Langdon, A. B. 1992, ApJ, 390,454

Kirk, J. G., \& Schneider, P. 1987, ApJ, 315, 425

Kirk, J. G., Guthmann, A. W., Gallant, Y. A, \& Achterberg, A. 2000, ApJ, 542, 235

Medvedev, M. V., \& Loeb, A. 1999, ApJ, 526, 697

Ostrowski, M. 1993, MNRAS, 264, 248

Peacock, J. A. 1981, MNRAS, 196, 135

Pohl, M., Lerche, I., \& Schlickeiser, R. 2001, in Proc. 27th Int. Cosmic Ray Conf., OG 2.3, 2713

Vietri, M. 2002, submitted 